\title{
Manipulation of Cooper Pair Entanglement in Hybrid Topological Josephson Junctions ${ }^{\dagger}$
}

\author{
Gianmichele Blasi * ${ }^{\mathbb{D}}$, Fabio Taddei, Vittorio Giovannetti and Alessandro Braggio \\ NEST, Scuola Normale Superiore and Istituto Nanoscienze-CNR, I-56126 Pisa, Italy; fabio.taddei@sns.it (F.T.); \\ vittorio.giovannetti@sns.it (V.G.); alessandro.braggio@nano.cnr.it (A.B.) \\ * Correspondence: gianmichele.blasi@sns.it \\ + Presented at the 11th Italian Quantum Information Science conference (IQIS2018), Catania, Italy, \\ 17-20 September 2018.
}

Received: 22 November 2018; Accepted: 10 May 2019; Published: 16 September 2019

\begin{abstract}
The non-local manipulation of spin-entangled states by means of local gating in two parallel 2D topological insulators properly connected to two superconducting electrodes is studied. We calculate analytically the current-phase relationship of the Josephson current making use of the scattering matrix approach and we identify the various local and non-local scattering mechanisms. We show that the Josephson critical current, remarkably, allows a direct quantification of the entanglement manipulation.
\end{abstract}

Keywords: electronic transport in mesoscopic systems; entanglement production and manipulation; andreev reflection; hybrid topological Josephson junctions

\section{The Setup}

In a Josephson system with ideal interfaces and rigid boundary conditions, the phase difference $\phi=\phi_{R}-\phi_{L}$ induces a stationary Josephson current. Microscopically it originates from Andreev reflection processes that describe the transfer of Cooper pairs (CPs) at the interfaces between the superconductors and the weak link. In a single 2D TI-plane sandwiched between two conventional s-wave superconductors, CPs can only be injected or absorbed locally on a specific edge. In order to have crossed Andreev reflections (CARs) among different edges one needs to consider two edge states with the same helicity. Specifically in Ref. [1] we focused on the architecture depicted in Figure 1 where a Josephson junction is obtained by sandwiching two planes of 2D TIs in between two s-wave superconductors. This system allows for CAR processes if the distance $W$ between the two TI-planes is comparable with the coherence length $\xi$. Moreover, the properties of the edge modes can be tuned through the application of "local fields": on the lower TI, we consider the application of a time-reversal Rashba-like spin-orbit (SO) field realized by placing a constant gate affecting the dynamical phase of the carriers along the edge [2]; while, on the upper TI plane, we assumed a local Zeeman-like (B) field which is time-reversal breaking and can be realized exploiting the Doppler shift effect induced by a magnetic flux through the junction (see Figure 1) [3,4]. 


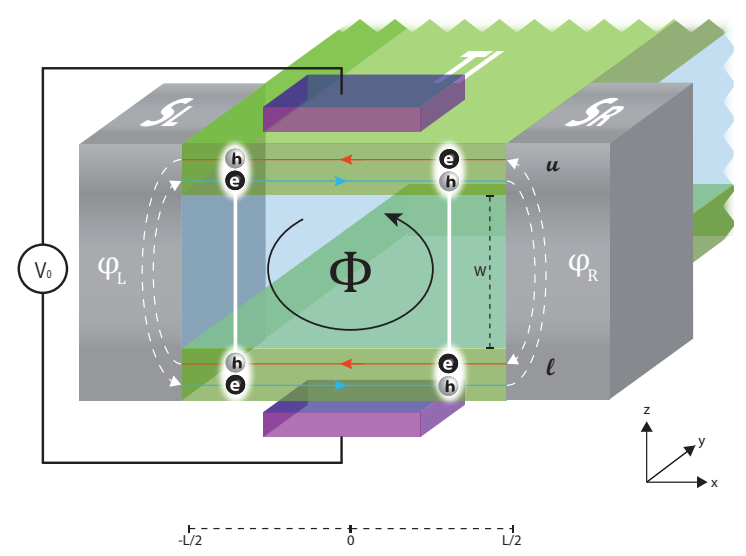

Figure 1. Schematic representation of the setup: it consists of an heterostructure (say CdTe-HgTe) grown along the $z$-axis resulting in two layers of TIs. In gray are depicted the superconductors $\left(S_{L / R}\right)$ while in green the TI planes. The arrowed blue (red) solid lines represent the $1 \mathrm{D}$ helical edge states with spin $\uparrow(\downarrow)$. On the frontal side, in the $x$-z-plane of the scheme, are depicted two CAR processes where $\mathrm{CP}$ are non-locally splitted. The application of the Rashba and Zeeman-like fields due to the presence of side gates and the induced magnetic flux $\Phi$. Such a fields act in terms of the unitary operators $\mathcal{U}_{\mathrm{B}}^{\mathrm{u}}\left(\theta_{\mathrm{B}}\right)$ and $\mathcal{U}_{\mathrm{SO}}^{\ell}\left(\theta_{\mathrm{SO}}\right)$ (see text) on the upper and lower edges respectively.

\section{The Model}

Following the scattering approach [5-7], we describe the setup of Figure 1 by means of its scattering matrix, which relates the incoming electron or hole amplitudes impinging onto the interfaces with the superconductors with the outgoing electron or hole amplitudes $[8,9]$. The scattering matrix of the Andreev processes occurring on the left L (right R) TI-S interface, in the $\mathrm{u}-\mathrm{l}$ space, can be written as $\left[\mathbb{1}\left|\Lambda_{\mathrm{L}(\mathrm{R})}\right|+i \sigma_{x}\left|X_{\mathrm{L}(\mathrm{R})}\right|\right] e^{i \phi_{\mathrm{L}(\mathrm{R})}}$, with $\sigma_{x}$ the Pauli matrix and $\Lambda_{\mathrm{L}(\mathrm{R})}$ and $X_{\mathrm{L}(\mathrm{R})}$ representing respectively the amplitude for the LAR and CAR events (these terms being related by the unitarity conditions $\left.\left|\Lambda_{L(R)}\right|^{2}+\left|X_{L(R)}\right|^{2}=1\right)$. In writing the above expression we neglected the presence of the edge modes running in the backside part of the device of Figure 1. This is justified by assuming that the size of the TI in the $y$-direction is much larger than the coherence length $\ell_{\phi}$ or having intentionally broken superconducting coherence introducing a dephasing source along those edges, e.g. by adding a floating metal pad over those edge modes which will induce electron decoherence. So backside modes decrease the supercurrent introducing an effective loss parameter $\eta \in[0,1]$ where $\eta=0$ represents lossless regime. The action of the two local fields along the edge length $L$ is described, in the spin space, by the following unitary operators: $\mathcal{U}_{\mathrm{B}}^{\mathrm{u}}\left(\theta_{\mathrm{B}}\right)=\exp \left[i \theta_{\mathrm{B}} \sigma_{z} / 2\right]$ and $\mathcal{U}_{\mathrm{SO}}^{\ell}\left(\theta_{\mathrm{SO}}\right)=\exp \left[i \operatorname{sign}\left(p_{x}\right) \theta_{\mathrm{SO}} \sigma_{z} / 2\right]$, where the $z$-axis is the quantization axis of both the TI planes and $p_{x}$ is the momentum of electrons moving in the $x$-direction. The manipulation angles $\theta_{B}=4 \pi B W L / \Phi_{0} \equiv 4 \pi \Phi / \Phi_{0}$ and $\theta_{S O}=2 e V_{0} L / \hbar v_{F}$ can be obtained by exploiting the Doppler shift effect and a constant gate respectively (see Figure 1).

\section{Local Fields Selective Action}

The local action of the fields $\mathcal{U}_{\mathrm{B}}\left(\theta_{\mathrm{B}}\right)$ and $\mathcal{U}_{\mathrm{SO}}\left(\theta_{\mathrm{SO}}\right)$ operates independently and selectively on the local and non-local components of the Josephson current. Before solving explicitly the transport equations of the model, a preliminary evidence of this fact is obtained via an heuristic argument applied to the simplified scenario where LARs are absent (i.e., $\Lambda_{\mathrm{L}(\mathrm{R})}=0$ ). Under this circumstance the non-local emission of a $\mathrm{CP}$ from a superconducting electrode, say $\mathrm{S}_{\mathrm{L}}$, results in the formation of a spin-entangled $\mathrm{CP}$ state, which arises from two superimposed CAR processes. In the first one, a spin- $\downarrow$ hole propagating in the lower edge gets reflected into an spin- $\uparrow$ electron in the upper edge, while in the second one, a spin- $\downarrow$ hole propagating in the upper edge gets reflected into an spin- $\uparrow$ electron in the lower edge [see Figure 1]. Such spin-entangled state could 
be represented as $|C\rangle=\left(\left|e_{u}^{\uparrow} h_{\ell}^{\downarrow}\right\rangle-\left|h_{u}^{\downarrow} e_{\ell}^{\uparrow}\right\rangle\right) / \sqrt{2}$, where the minus sign recall the fact that the CP is in a spin-singlet state of s-wave Ss. The action of the local fields on $|C\rangle$ results in the state: $e^{i \frac{\theta_{B}}{2}}\left(e^{-i \theta_{\mathrm{SO}} / 2}\left|e_{u}^{\uparrow} h_{\ell}^{\downarrow}\right\rangle-e^{+i \theta_{\mathrm{SO}} / 2}\left|h_{u}^{\downarrow} e_{\ell}^{\uparrow}\right\rangle\right) / \sqrt{2}$. This expression shows that while the B-field introduces only a global phase, that can be reabsorbed with a gauge transformation, the SO-field modifies the entanglement symmetry of the non-local $\mathrm{CP}$ state $|C\rangle$ by introducing a relative phase factor $\exp \left(i \theta_{\mathrm{SO}}\right)$, without altering its entanglement content. In particular, if $\theta_{\mathrm{SO}}=\pi$ the non-local spin-singlet $\mathrm{CP}$ changes into a spin-triplet one, thus giving rise to a mismatch with respect to the intrinsic CPs singlet symmetry of the electrodes, thus hindering the Josephson coupling. In view of this fact, in the absence of LAR processes, one hence expects the Josephson current to be proportional to the quantity $\mathcal{C}=\left|\left\langle C\left|\mathcal{U}_{S O}\left(\theta_{S O}\right)\right| C\right\rangle\right|=\left|\cos \left(\theta_{S O} / 2\right)\right|$, which measures the degree of change of the symmetry of the entangled $\mathrm{CP}$.

\section{Results}

To set the above observations on firm ground, in Ref. [1] we computed the critical current $J_{c}$ of the system, i.e., the maximum $J_{c}=\max _{\phi}\{|J(\phi)|\}$ of the Josephson current $J(\phi)$. Using the scattering formalism [8-10] in the short junction limit, i.e., when $L \ll \xi$, the latter can be expressed as $J(\phi)=-\frac{2 e}{\hbar} \sum_{p} \tanh \left(\epsilon_{p} / 2 k_{B} T\right) \frac{d \epsilon_{p}}{d \phi}$, where $\epsilon_{p}$ are Andreev bound state energies obtained solving the self-consistent secular problem [10]. Remarkably, we find that for the proposed setup $J_{\mathcal{c}}$ can be written in the following compact form

$$
J_{\mathrm{C}}=\alpha(\eta, T)|\Gamma|+\beta(\eta, T),
$$

where $\Gamma=\cos \left(\theta_{\mathrm{SO}} / 2\right)\left|X_{L}\right|\left|X_{R}\right|+\cos \left(\theta_{B} / 2\right)\left|\Lambda_{L}\right|\left|\Lambda_{R}\right|$ depends only on the Andreev reflection amplitudes $X_{L}$ and $X_{R}$, and on the local fields strengths $\theta_{B}$ and $\theta_{S O}$, while the prefactor $\alpha$ and the off-set $\beta$ depend only on the temperature $T$ and on the losses $\eta$.

By close inspection of Equation (1) one obseves that the critical current in a pure LAR scenario results as a constant function of the manipulation angle $\theta_{S O}$. Any deviation from this behaviour is an unequivocal signature of the presence of CAR processes at both the interfaces with the superconductors. Furthermore it is worth noting that one can always nullify the contributions to the current of the LAR processes by properly tuning the external Zeeman-like field to have $\theta_{B}=\pi$ : in this way one can single out the component of the current arising from the purely CAR processes.

Hereafter we claim that the dependence on $\theta_{S O}$ of $J_{c}$ such as determined in Equation (1) indicates that the critical current reflects the entanglement symmetry manipulation due to the action of the SO field. We first notice that the critical current, resulting from Andreev bound states within the junction, can be seen as consisting of the sum of contributions arising from multiple Andreev reflection processes. In the only-CAR regime one can identify, for any values of $\eta$, two classes of processes: the ones corresponding to Cooper pairs which traverse the junction back and forth an even number of times and the processes which traverse the junction an odd number of times. For the even class, the singlet symmetry is not modified by the effect of the SO-field, since the backward time-reversed propagation cancels the SO-field-induced phase taken during the forward propagation. The spin entanglement symmetry is instead changed only for the odd class processes. This suggests that, at zero temperature and without losses $(\eta=0)$, the odd class processes contribute to the critical current with the term $\mathcal{C}=\left|\left\langle C\left|\mathcal{U}_{S O}\left(\theta_{S O}\right)\right| C\right\rangle\right|=\left|\cos \left(\theta_{S O} / 2\right)\right|$ in units of $J_{0}=4 \Delta_{0} \frac{e}{\hbar}$. At the same time the even class is independent of $\theta_{S O}$ and contributes to the current with the constant value $J_{0}$ (this give rise to the off-set $\beta$ in Equation (1)). Indeed, the critical current can be written as $J_{\mathrm{c}}=J_{0}(1+\mathcal{C})$. In particular, at $\theta_{S O}=\pi$ the entanglement symmetry of the non-local electronic state is changed into triplet in half of the processes (the odd ones) and is left singlet in the other half (the even ones). As a result, the non-local electronic state is an equal weighted mixture of singlet and triplet states. This interpretation is actually 
corroborated by the fact that when only lowest order processes contribute, i.e., in the single-shot regime occurring when $\eta \simeq 1$, the critical current in the leading term of $(1-\eta)$, takes the following form

$$
J_{c}=\frac{e \Delta_{0}}{\hbar}\left|\cos \left(\theta_{S O} / 2\right)\right|(1-\eta)^{4}+\mathcal{O}\left((1-\eta)^{6}\right)
$$

The lowest order $(1-\eta)^{4}$ accounts for the single shot CP process, where the CP is splitted at one barrier, taking an $(1-\eta)^{2}$ factor, and another factor when it recombines on the other barrier. In this case one finds a complete blocking of the critical current in correspondence to $\theta_{S O}=\pi$. Previous results show that in the limit of single shot regime the action of the local SO field on the single CP returns exactly the expected entanglement manipulation signature $\mathcal{C}$. Furthermore Equation (1) clarifies that standard critical currents allows one to access experimentally the product $\left|X_{R}\right|\left|X_{L}\right|$ which determines the relative weight between the LAR and CAR processes.

\section{Conclusions}

In this paper we have review the setup proposed in Ref. [1] which makes use of helical edge states of a TI to spatially separate the two electrons composing a CP of a superconductor, thus enabling to manipulate the symmetry of a maximally entangled state. In particular we have shown that a measurable signature of this effect is provided by the critical current of the model.

\section{References}

1. Blasi, G.; Taddei, F.; Giovannetti, V.; Braggio, A. Manipulation of Cooper pair entanglement in hybrid topological Josephson junctions. arXiv 2018, arXiv:1808.09709.

2. Xiao, X.; Liu, Y.; Liu, Z.; Ai, G.; Yang, S.A.; Zhou, G. All-electric spin modulator based on a two-dimensional topological insulator. Appl. Phys. Lett. 2016, 108, 032403.

3. Tkachov, G.; Burset, P.; Trauzettel, B.; Hankiewicz, E. Quantum interference of edge supercurrents in a two-dimensional topological insulator. Phys. Rev. B 2015, 92, 045408, doi:10.1103/PhysRevB.92.045408.

4. Sothmann, B.; Giazotto, F.; Hankiewicz, E.M. High-efficiency thermal switch based on topological Josephson junctions. New J. Phys. 2017, 19, 023056.

5. Büttiker, M. Four-Terminal Phase-Coherent Conductance. Phys. Rev. Lett. 1986, 57, 1761-1764, doi:10.1103/ PhysRevLett.57.1761.

6. Büttiker, M. Scattering theory of current and intensity noise correlations in conductors and wave guides. Phys. Rev. B 1992, 46, 12485-12507, doi:10.1103/PhysRevB.46.12485.

7. Lambert, C.J.; Raimondi, R. Phase-coherent transport in hybrid superconducting nanostructures. J. Phys. Condens. Matter 1998, 10, 901.

8. Beenakker, C.W.J. Universal limit of critical-current fluctuations in mesoscopic Josephson junctions. Phys. Rev. Lett. 1991, 67, 3836-3839, doi:10.1103/PhysRevLett.67.3836.

9. Beenakker, C. Three "universal" mesoscopic Josephson effects. In Transport Phenomena in Mesoscopic Systems; Springer: Berlin/Heidelberg, Germany, 1992; pp. 235-253.

10. Beenakker, C.W.J.; Emary, C.; Kindermann, M.; van Velsen, J.L. Proposal for Production and Detection of Entangled Electron-Hole Pairs in a Degenerate Electron Gas. Phys. Rev. Lett. 2003, 91, 147901, doi:10.1103/ PhysRevLett.91.147901.

(C) 2019 by the authors. Licensee MDPI, Basel, Switzerland. This article is an open access article distributed under the terms and conditions of the Creative Commons Attribution (CC BY) license (http://creativecommons.org/licenses/by/4.0/). 